\title{
NON-STICKING OSCILLATION FORMULAE FOR COULOMB FRICTION UNDER HARMONIC LOADING
}

\author{
H.-K. HONG \\ Department of Civil Engineering, Taiwan University, Taipei, Taiwan
}

AND

C.-S. LIU

Department of Mechanical and Marine Engineering, Taiwan Ocean University, Keelung, Taiwan. E-mail: csliu@earth.me.ntou.edu.tw

(Received 27 July 2000, and in final form 18 December 2000)

\begin{abstract}
In this paper, a new estimate for periodic non-sticking (i.e., zero stop per cycle) solutions is presented for the steady state responses of the Coulomb friction oscillator subjected to harmonic loading. Compared with the Den Hartog (1931 Transactions of the American Society of Mechanical Engineers 53, 107-115 [1]) estimate, the new estimate leads to the same formulae for the maximum displacement and its time lag, but only the new estimate offers the closed-form formulae for the maximum velocity and its time lag. More importantly, a simple formula is derived for estimating the minimum driving force amplitude needed to prevent an oscillating object from sticking to the friction surface on which it slides. The validity of the assumptions made for the new estimate and the accuracy of the formulae developed are confirmed by comparing with the exact solutions (Hong and Liu 2000 Journal of Sound and Vibration 229, 1171-1192 [2]). It is also found that there exists the best driving force amplitude for maximum dissipation efficiency.
\end{abstract}

(C) 2001 Academic Press

\section{INTRODUCTION}

Friction is present in all machines and structures incorporating parts with relative motion. In some instances the motion may be induced by vibration and may result in a stick-slip interaction. Friction is also the principal source of dissipation, which may be a desirable property in some machinery and building applications, such as brakes and base isolators. To prevent an oscillating object from sticking to the friction surface on which it slides is an important issue in engineering sciences and industrial applications; see, e.g., references [3, 4]. As early as in 1931 Den Hartog [1] was able to simplify the frictional oscillation problem, presenting a periodic non-sticking (i.e., zero stop per cycle) solution of the steady state responses of the Coulomb friction oscillator subjected to simple harmonic loading. The same problem was treated by using a number of techniques, such as various time domain numerical integration methods [5],various phase plane methods $[1,6,7]$, the incremental harmonic balance method [8], equivalent linearization method [9, 10], etc.

Den Hartog's formulae are simple yet significant in the sense that they are a handy tool for the engineer's judgement. The significance is that it effectively reveals the factors of influence. The simplicity comes from a crucial assumption typical of the phase plane methods on the phase plane which is close to reality on many occasions. The study based 


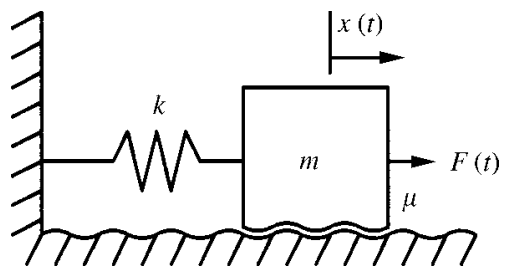

Figure 1. The mass-spring-friction oscillator, where the friction refers to Coulomb's perfect dry friction between the mass and the ground surface.

upon the exact solutions (see, e.g., references $[2,5]$ ) made clear the prevalence of the motion of zero stop per cycle in the steady state, thus confirming the significance of the formulae. However, Den Hartog's method could only estimate the maximum displacement and its phase lag (and hence its time lag), but failed to estimate the maximum velocity and its time lag. Moreover, an even more important issue is to develop a simple yet effective formula for predicting the minimum driving force amplitude to prevent an oscillating object from sticking to the friction surface. This issue has not yet been solved in the literature including Den Hartog [1], and several recent articles studying the base isolation behavior with friction [11-17].

The present paper thus aims to extend Den Hartog's work, developing simple formulae for use in engineering applications. The derived formulae are exact solutions of an approximate theory, the accuracy of which is also examined critically in the paper.

To begin with, we show the governing equation of the harmonically excited oscillator as follows (see Figure 1):

$$
m \ddot{x}(t)+r(t)=p_{0} \sin \omega_{d} t,
$$

where $p_{0}$ and $\omega_{d}$ are the amplitude and angular frequency, respectively, of the driving harmonic force; $t$ is time; a superposed dot represents time differentiation; $x, \dot{x}, \ddot{x}$ and $m$ denote the position co-ordinate, velocity, acceleration and mass, respectively, of the body of the oscillator. The co-ordinate $x$ is chosen such that $x=0$ is the static equilibrium position. In the Coulomb friction oscillator the constitutive force $r$ is composed of the spring force $r_{b}$ and the friction force $r_{a}$, with two prescribed positive constants: the spring stiffness $k$ and the friction bound $r_{y}$. That is, $r=r_{a}+r_{b}, r_{b}=k x$, and $\left|r_{a}\right| \leqslant r_{y}$.

If the body of the oscillator is subjected to a harmonic base translation $u_{g}(t)=u_{g 0} \sin \omega_{d} t$, $u_{g 0}$ being the amplitude of the periodic base excitation, the equation of motion may be recast to the same equation (1) by setting $p_{0}=k u_{g 0}$ and re-designating $x, \dot{x}, \ddot{x}$ to be relative to the base.

\section{A NEW ESTIMATE OF THE STEADY STATE RESPONSES}

Let us consider a steady state non-sticking cycle and assume the phase curve of such a cycle in the phase plane $(x, \dot{x})$ to be symmetrical with respect to the origin as shown in Figure 2. Therefore, the phase curve is closed in the phase plane and it suffices to consider only one half of the curve, say the right branch. (One can also choose the left branch and obtain the same results as ours, but neither the upper branch nor the lower branch each of which gives the Den Hartog results.) Let us denote by $\Delta_{0}$ and $V_{0}$ the maximum displacement and velocity, respectively, of the steady motion. Here, we do not assume that the points of the maximum and minimum velocities are located on the $\dot{x}$-axis, and allow 


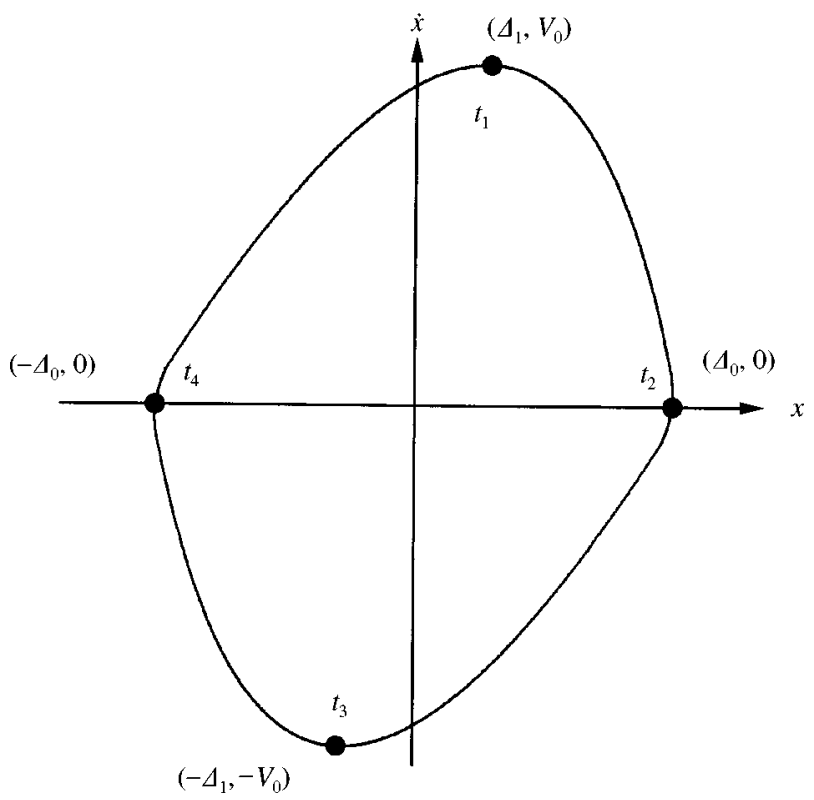

Figure 2. A typical steady state non-sticking oscillatory response in the phase plane, where $t_{1}, t_{2}, \Delta_{0}, V_{0}$ and $\Delta_{1}$ are to be determined.

these two points to deviate from the $\dot{x}$-axis with an unknown deviation $\Delta_{1}$ as shown in Figure 2. In view of the periodicity of the input and the symmetry assumption, we further assume $(x, \dot{x})$ equal to $\left(\Delta_{1}, V_{0}\right),\left(\Delta_{0}, 0\right),\left(-\Delta_{1},-V_{0}\right)$ and $\left(-\Delta_{0}, 0\right)$ at time $t_{1}, t_{2}, t_{3}$ and $t_{4}$, respectively, with

$$
t_{3}=t_{1}+\frac{\pi}{\omega_{d}}, \quad t_{4}=t_{2}+\frac{\pi}{\omega_{d}},
$$

where $t_{2}$ is the time at which $x(t)$ reaches its maximum value in a steady state cycle, while $t_{4}$ is the time at which $x(t)$ obtains its minimum value; similarly, $t_{1}$ is the time at which $\dot{x}(t)$ reaches its maximum value, while $t_{3}$ is the time at which $\dot{x}(t)$ obtains its minimum value.

We now determine the five parameters, namely the maximum displacement $\Delta_{0}$, the maximum velocity $V_{0}$, the deviation $\Delta_{1}$ and the time instants $t_{1}$ and $t_{2}$ so as to match the exact solutions of the steady state responses. In the time interval $t_{1} \leqslant t \leqslant t_{2}$ the steady state solution of equation (1) is

$$
x_{1}(t)=A \sin \omega_{d} t-B+a_{1} \sin \omega_{n}\left(t-t_{1}\right)+b_{1} \cos \omega_{n}\left(t-t_{1}\right) .
$$

Here

$$
A:=\frac{p_{0}}{k\left(1-\Omega^{2}\right)}, \quad B:=\frac{\left(1-\Omega^{2}\right) A}{\alpha}, \quad \omega_{n}:=\sqrt{\frac{k}{m}}, \quad \alpha:=\frac{p_{0}}{r_{y}}, \quad \Omega:=\frac{\omega_{d}}{\omega_{n}},
$$

in which $\omega_{n}$ is the natural frequency, $\alpha$ the force amplitude ratio, and $\Omega$ the frequency ratio. $B=r_{y} / k$ may be viewed as a virtual friction displacement at which the slider just sustains enough spring force to overcome the friction bound and to start the sliding. Similarly, in the 
time interval $t_{2} \leqslant t \leqslant t_{3}$ we have

$$
x_{2}(t)=A \sin \omega_{d} t+B+a_{2} \sin \omega_{n}\left(t_{3}-t\right)+b_{2} \cos \omega_{n}\left(t_{3}-t\right) .
$$

The four constants $a_{1}, b_{1}, a_{2}$ and $b_{2}$ can be determined using the following four conditions:

$$
x_{1}\left(t_{1}\right)=\Delta_{1}, \quad x_{2}\left(t_{3}\right)=-\Delta_{1}, \quad \dot{x}_{1}\left(t_{1}\right)=V_{0}, \quad \dot{x}_{2}\left(t_{3}\right)=-V_{0} .
$$

As a result we obtain

$$
\begin{aligned}
x_{1}(t)= & A\left[\sin \omega_{d} t-\sin \omega_{d} t_{1} \cos \omega_{n}\left(t-t_{1}\right)-\Omega \cos \omega_{d} t_{1} \sin \omega_{n}\left(t-t_{1}\right)\right] \\
& +\left(B+\Delta_{1}\right) \cos \omega_{n}\left(t-t_{1}\right)-B+\frac{V_{0}}{\omega_{n}} \sin \omega_{n}\left(t-t_{1}\right), \\
x_{2}(t)= & A\left[\sin \omega_{d} t-\sin \omega_{d} t_{3} \cos \omega_{n}\left(t-t_{3}\right)-\Omega \cos \omega_{d} t_{3} \sin \omega_{n}\left(t-t_{3}\right)\right] \\
& -\left(B+\Delta_{1}\right) \cos \omega_{n}\left(t-t_{3}\right)+B-\frac{V_{0}}{\omega_{n}} \sin \omega_{n}\left(t-t_{3}\right) .
\end{aligned}
$$

With equations (15), (16), (2) and (3) the four conditions

$$
\dot{x}_{1}\left(t_{2}\right)=0, \quad \dot{x}_{2}\left(t_{2}\right)=0, \quad x_{1}\left(t_{2}\right)=\Delta_{0}, \quad x_{2}\left(t_{2}\right)=\Delta_{0},
$$

become the following four equations:

$$
\begin{aligned}
& A \Omega \cos \omega_{d} t_{2}-\left(B+\Delta_{1}-A \sin \omega_{d} t_{1}\right) \sin \omega_{n}\left(t_{2}-t_{1}\right)+\rho \cos \omega_{n}\left(t_{2}-t_{1}\right)=0, \\
& A \Omega \cos \omega_{d} t_{2}+\left(B+\Delta_{1}-A \sin \omega_{d} t_{1}\right)\left[\cos \pi_{1} \sin \omega_{n}\left(t_{2}-t_{1}\right)-\sin \pi_{1} \cos \omega_{n}\left(t_{2}-t_{1}\right)\right] \\
& \quad-\rho\left[\cos \pi_{1} \cos \omega_{n}\left(t_{2}-t_{1}\right)+\sin \pi_{1} \sin \omega_{n}\left(t_{2}-t_{1}\right)\right]=0, \\
& A \sin \omega_{d} t_{2}+\left(B+\Delta_{1}-A \sin \omega_{d} t_{1}\right) \cos \omega_{n}\left(t_{2}-t_{1}\right)+\rho \sin \omega_{n}\left(t_{2}-t_{1}\right)=\Delta_{0}+B, \\
& A \sin \omega_{d} t_{2}-\left(B+\Delta_{1}-A \sin \omega_{d} t_{1}\right)\left[\cos \pi_{1} \cos \omega_{n}\left(t_{2}-t_{1}\right)+\sin \pi_{1} \sin \omega_{n}\left(t_{2}-t_{1}\right)\right] \\
& \quad-\rho\left[\cos \pi_{1} \sin \omega_{n}\left(t_{2}-t_{1}\right)-\sin \pi_{1} \cos \omega_{n}\left(t_{2}-t_{1}\right)\right]=\Delta_{0}-B .
\end{aligned}
$$

At the same time, letting $\ddot{x}_{2}\left(t_{1}+\pi / \omega_{d}\right)=0$ for the minimum velocity at $t=t_{3}=t_{1}+\pi / \omega_{d}$, we obtain

$$
A\left(\Omega^{2}-1\right) \sin \omega_{d} t_{1}+B+\Delta_{1}=0
$$

where

$$
\pi_{1}:=\frac{\pi}{\Omega}, \quad \rho:=\frac{V_{0}}{\omega_{n}}-A \Omega \cos \omega_{d} t_{1}
$$

Equations (21)-(25) taken together can be used to determine the five unknowns $t_{1}, t_{2}, V_{0}, \Delta_{0}$ and $\Delta_{1}$. 


\section{VALIDITY OF THE ASSUMPTIONS}

Until now we have not yet checked the validity of the symmetry assumption [cf. equations (2), (3), (11)-(14) and (17)-(20) in the above, and also the assumptions used by Den Hartog [1]]. In order to account of such problems let us define four error measures as follows:

$$
\begin{aligned}
& \text { Error } 1:=|\max x(t)+\min x(t)| / \max x(t), \\
& \text { Error } 2:= \begin{cases}\left(t_{4}-t_{2}-\pi / \omega_{d}\right) / T & \text { if } t_{4} \geqslant t_{2}, \\
\left(t_{2}-t_{4}-\pi / \omega_{d}\right) / T & \text { if } t_{2}>t_{4},\end{cases} \\
& \text { Error } 3:=\mid \begin{array}{ll}
\max \dot{x}(t)+\min \dot{x}(t) \mid / \max \dot{x}(t),
\end{array} \\
& \text { Error } 4:= \begin{cases}\left(t_{3}-t_{1}-\pi / \omega_{d}\right) / T & \text { if } t_{3} \geqslant t_{1}, \\
\left(t_{1}-t_{3}-\pi / \omega_{d}\right) / T & \text { if } t_{1}>t_{3},\end{cases}
\end{aligned}
$$

in which $T:=2 \pi / \omega_{d}$ is the period of the driving force.

First, the exact solutions in the steady state were derived by Hong and Liu [2]:

$$
\begin{aligned}
x(t)= & x\left(t_{i}\right)+\frac{\dot{x}\left(t_{i}\right)}{\omega_{n}} \sin \omega_{n}\left(t-t_{i}\right)-\frac{r\left(t_{i}\right)}{k}\left[1-\cos \omega_{n}\left(t-t_{i}\right)\right] \\
& +A\left[\sin \omega_{d} t-\cos \omega_{n}\left(t-t_{i}\right) \sin \omega_{d} t_{i}-\Omega \sin \omega_{n}\left(t-t_{i}\right) \cos \omega_{d} t_{i}\right], \\
r(t)= & r\left(t_{i}\right)+k\left[x(t)-x\left(\mathrm{t}_{i}\right)\right],
\end{aligned}
$$

when time $t$ is large. Here, $t_{i}$ is the initial time, and $x\left(t_{i}\right), \dot{x}\left(t_{i}\right)$ and $r\left(t_{i}\right)$ are the initial displacement, initial velocity and initial constitutive force respectively. On the basis of the calculated values of the exact solutions, the four error measures were calculated for the frequency ratio $\Omega$ in the range $0 \cdot 6-2 \cdot 0$ and for different values of $1 / \alpha=0 \cdot 3,0 \cdot 4,0 \cdot 5$, wherein all the steady motions had zero stop per cycle [2]. The errors were found rather small, of the order of $10^{-7}-10^{-5}$ for most of the cases, but near the resonance point, that is $\Omega=1$, some peaks appeared and the errors were of the order of $10^{-4}-10^{-2}$ as shown in Figure 3. The results suggest that the assumption made for the new estimate (and the Den Hartog estimate) is acceptable.

\section{CLOSED-FORM SOLUTIONS OF THE MAXIMUM DISPLACEMENT}

Eliminating $A \Omega \cos \omega_{d} t_{2}$ from equations (21) and (22), and $A \sin \omega_{d} t_{2}$ from equations (23) and (24) we obtain

$$
\begin{gathered}
a \cos \omega_{n}\left(t_{2}-t_{1}\right)+b \sin \omega_{n}\left(t_{2}-t_{1}\right)=0, \\
-b \cos \omega_{n}\left(t_{2}-t_{1}\right)+a \sin \omega_{n}\left(t_{2}-t_{1}\right)=2 B,
\end{gathered}
$$

where

$$
\begin{aligned}
& a:=\rho\left(1+\cos \pi_{1}\right)+\left(B+\Delta_{1}-A \sin \omega_{d} t_{1}\right) \sin \pi_{1}, \\
& b:=\rho \sin \pi_{1}-\left(B+\Delta_{1}-A \sin \omega_{d} t_{1}\right)\left(1+\cos \pi_{1}\right) .
\end{aligned}
$$



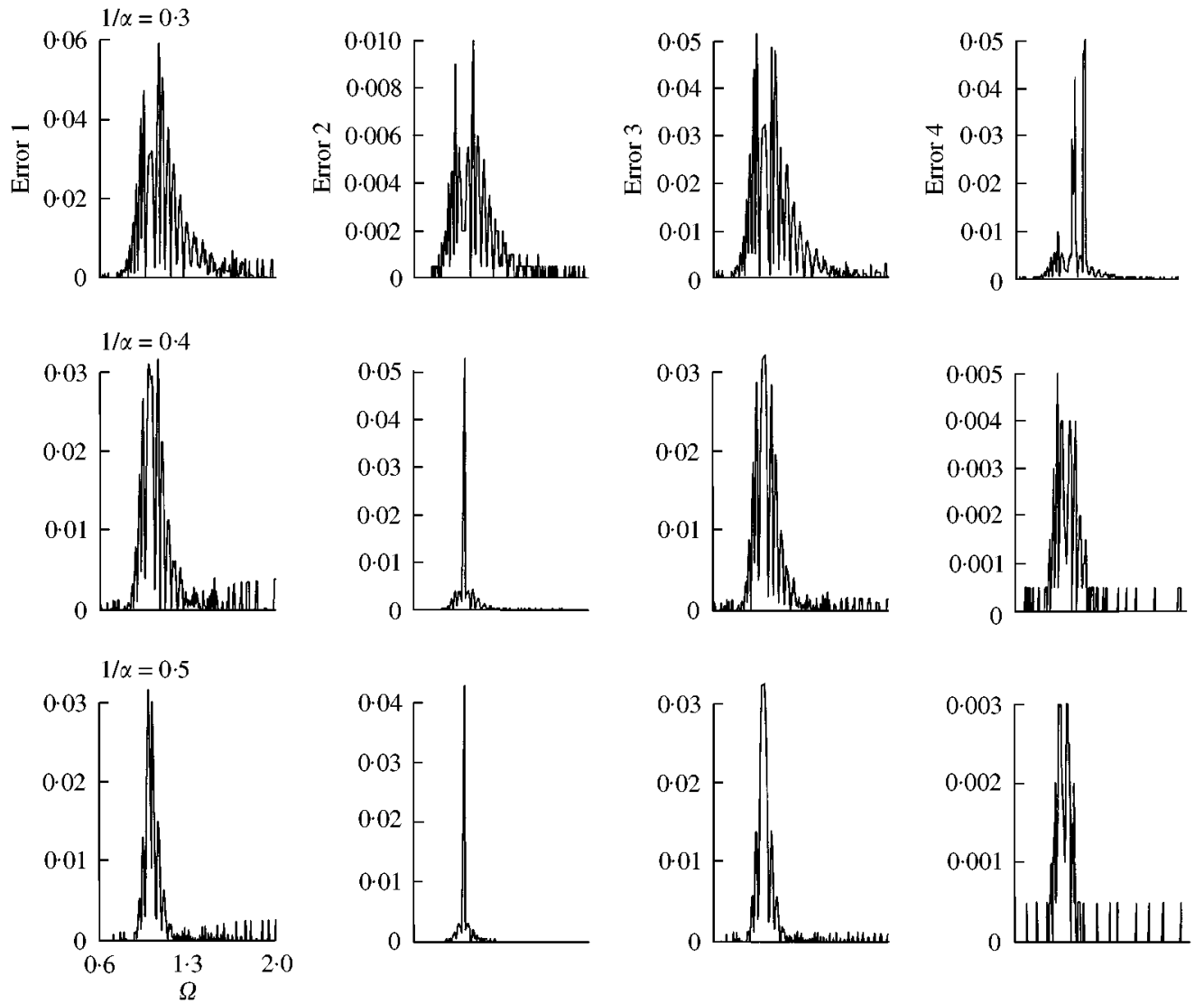

Figure 3. The errors of the assumptions.

From these equations it follows that

$$
a^{2}+b^{2}=2 \rho^{2}\left(1+\cos \pi_{1}\right)+2\left(B+\Delta_{1}-A \sin \omega_{d} t_{1}\right)^{2}\left(1+\cos \pi_{1}\right) .
$$

The solutions of equations (28) and (29) are

$$
\cos \omega_{n}\left(t_{2}-t_{1}\right)=\frac{-2 B b}{a^{2}+b^{2}}, \quad \sin \omega_{n}\left(t_{2}-t_{1}\right)=\frac{2 B a}{a^{2}+b^{2}},
$$

which together give

$$
a^{2}+b^{2}=4 B^{2} .
$$

From equations (32) and (35) it is obvious that

$$
\rho^{2}=\frac{2 B^{2}}{1+\cos \pi_{1}}-\left(B+\Delta_{1}-A \sin \omega_{d} t_{1}\right)^{2} .
$$

Employing this in equation (27) yields

$$
\frac{V_{0}}{\omega_{n}}=A \Omega \cos \omega_{d} t_{1}+\sqrt{\frac{2 B^{2}}{1+\cos \pi_{1}}-\left(B+\Delta_{1}-A \sin \omega_{d} t_{1}\right)^{2}} .
$$


Equations (33), (34), (30), (31) and (35) together lead to

$$
\begin{aligned}
& \cos \omega_{n}\left(t_{2}-t_{1}\right)=\frac{\left(1+\cos \pi_{1}\right)\left(B+\Delta_{1}-A \sin \omega_{d} t_{1}\right)-\rho \sin \pi_{1}}{2 B}, \\
& \sin \omega_{n}\left(t_{2}-t_{1}\right)=\frac{\rho\left(1+\cos \pi_{1}\right)+\left(B+\Delta_{1}-A \sin \omega_{d} t_{1}\right) \sin \pi_{1}}{2 B} .
\end{aligned}
$$

Substituting equations (37)-(39) into equation (23) gives

$$
A \sin \omega_{d} t_{2}=\Delta_{0}
$$

Similarly, substituting equations (37)-(39) into equation (21) leads to

$$
A \cos \omega_{d} t_{2}=\frac{B \sin \pi_{1}}{\Omega\left(1+\cos \pi_{1}\right)} .
$$

However, the substituting of equations (37)-(40) into equation (24), and of equations (37)-(39) and (41) into equation (22), give no new equation but equation (36) again.

From equations (40) and (41) it follows that

$$
\Delta_{0}=\sqrt{A^{2}-\left[\frac{B \sin \pi_{1}}{\Omega\left(1+\cos \pi_{1}\right)}\right]^{2}} .
$$

By equations (40) and (41) we have

$$
t_{2}=\frac{\pi}{2 \omega_{d}}+\frac{1}{\omega_{d}} \arccos \frac{\Delta_{0}}{A}
$$

Note that the value of $t_{2}$ is taken in the range $\pi /\left(2 \omega_{d}\right)<t_{2}<3 \pi /\left(2 \omega_{d}\right)$. The above equations give the closed-form solutions of $\Delta_{0}$ and $t_{2}$ respectively. Equation (42) can be non-dimensionalized such that the formula

$$
\frac{\Delta_{0}}{B}=\sqrt{\left(\frac{\alpha}{\left(1-\Omega^{2}\right.}\right)^{2}-\frac{\sin ^{2} \pi_{1}}{\Omega^{2}\left(1+\cos \pi_{1}\right)^{2}}}
$$

depends only on two dimensionless parameters $\Omega$ and $\alpha$. In Figure 4 , the results calculated by the above new estimate are compared with the exact responses in the steady state for several values of $\alpha$. For the calculations of the exact responses we fixed the values with $m=50 \mathrm{kN} \mathrm{s}^{2} / \mathrm{m}, r_{y}=5 \mathrm{kN}, \omega_{d}=2 \pi \mathrm{rad} / \mathrm{s}$, and the other parameters were then computed by $\omega_{n}=\omega_{d} / \Omega, k=m \omega_{n}^{2}$, and $p_{0}=\alpha r_{y}$. On the other hand, for the estimation done here the parameters $\Omega$ and $\alpha$ were restricted to render the oscillator non-sticking. It can be seen that the new estimate gives a very accurate estimation of the maximum displacement for all $\alpha$ investigated. The precise criterion for non-sticking will be derived in section 7 . 

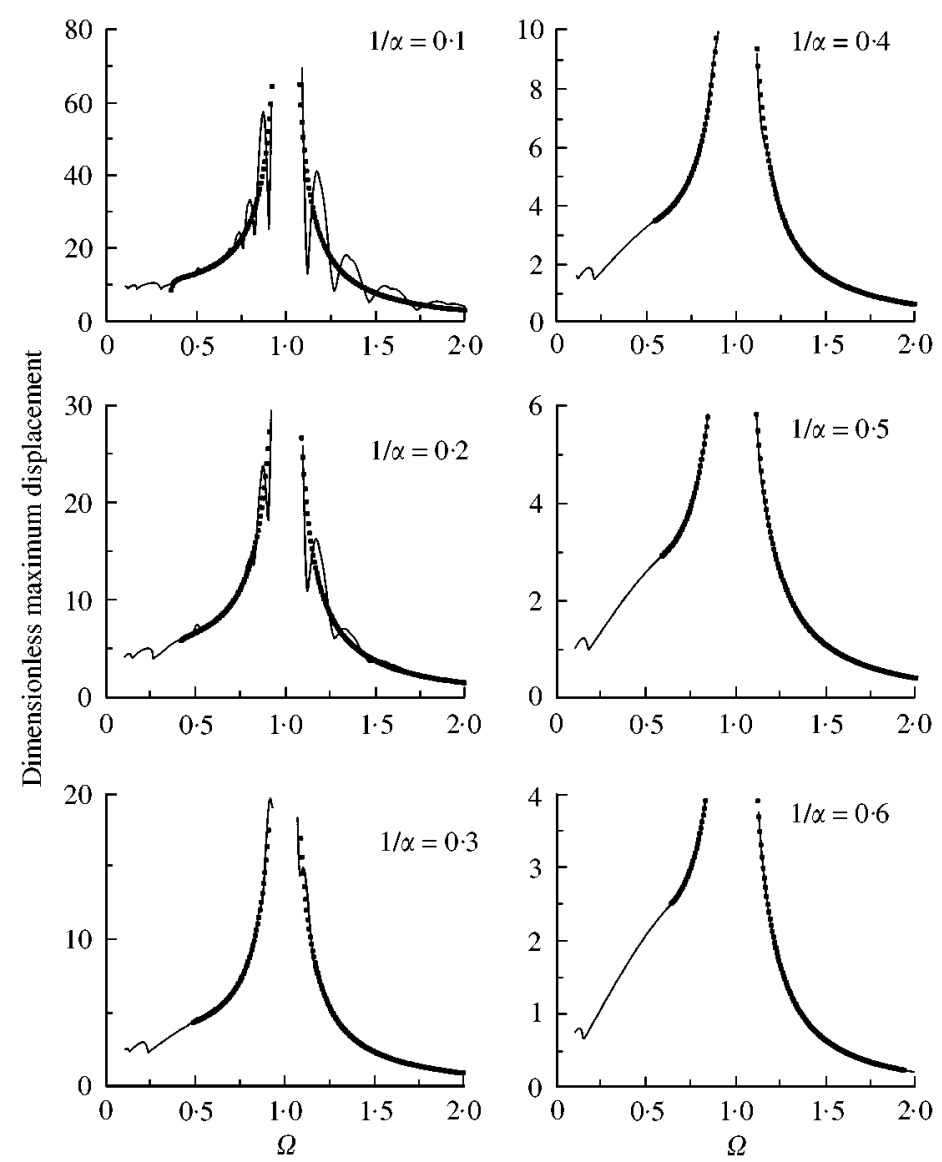

Figure 4. The comparison of the dimensionless maximum displacement between the exact solution and the new estimate for six values of $1 / \alpha$ : —, exact; $\mathbf{\square}$, estimated.

\section{CLOSED-FORM SOLUTIONS OF THE MAXIMUM VELOCITY}

Combining equations (38) and (39) gives

$$
\begin{gathered}
B\left(1+\cos \pi_{1}\right) \cos \omega_{n}\left(t_{2}-t_{1}\right)+B \sin \pi_{1} \sin \omega_{n}\left(t_{2}-t_{1}\right) \\
\quad=\left(B+\Delta_{1}\right)\left(1+\cos \pi_{1}\right)-A\left(1+\cos \pi_{1}\right) \sin \omega_{d} t_{1} .
\end{gathered}
$$

According to equation (25), we replace the term $B+\Delta_{1}$ by $A\left(1-\Omega^{2}\right) \sin \omega_{d} \mathrm{t}_{1}$ so that

$$
B\left(1+\cos \pi_{1}\right) \cos \omega_{n}\left(t_{2}-t_{1}\right)+B \sin \pi_{1} \sin \omega_{n}\left(t_{2}-t_{1}\right)+A \Omega^{2}\left(1+\cos \pi_{1}\right) \sin \omega_{d} t_{1}=0
$$

On the other hand, equation (25) can be written as

$$
\sin \omega_{d} t_{1}=\frac{1}{\alpha}+\frac{k}{p_{0}} \Delta_{1}
$$



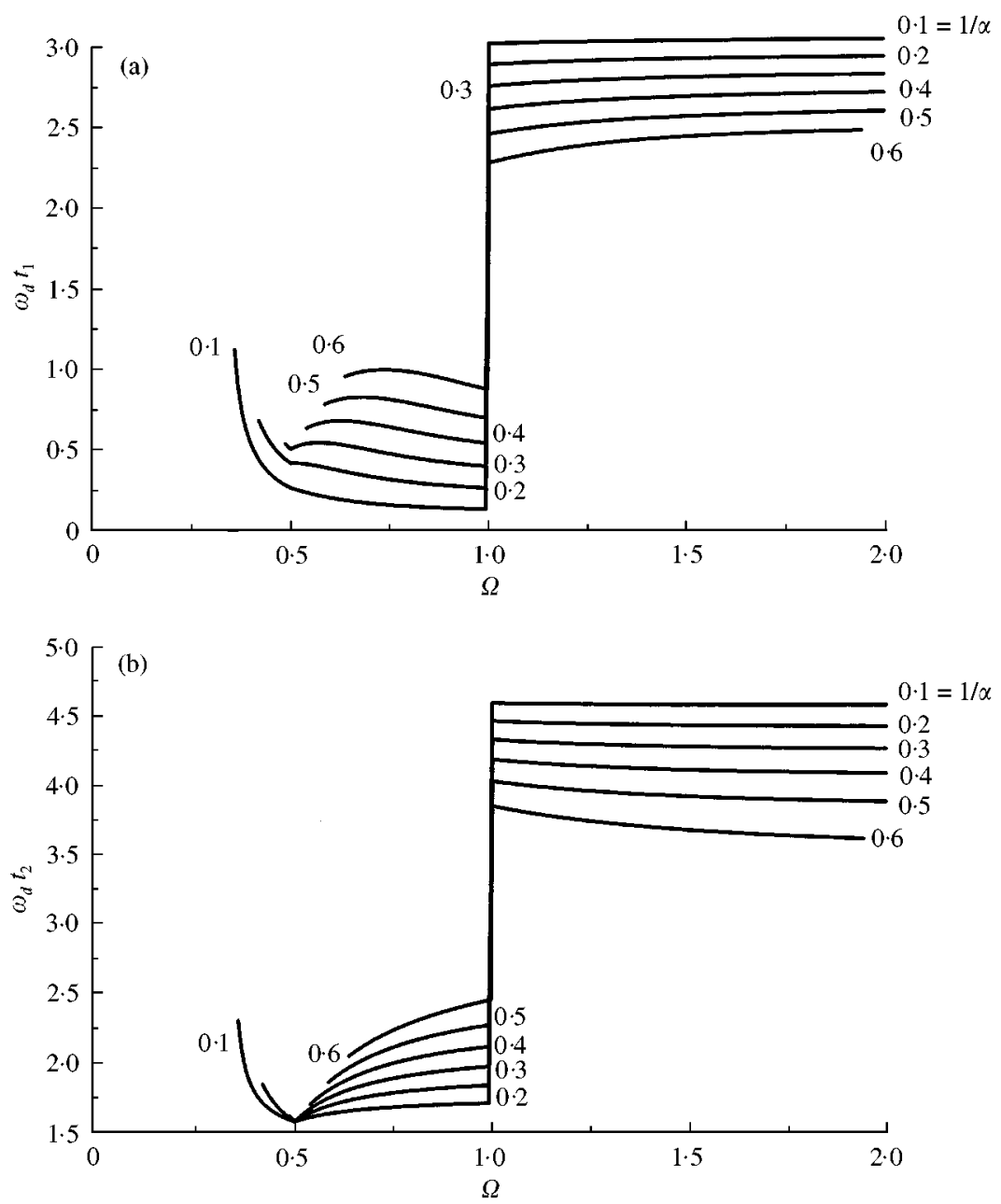

Figure 5. The two phase lags $\omega_{d} t_{1}$ and $\omega_{d} t_{2}$ for six values of $1 / \alpha$.

by equations (5)-(9). Substituting the above equation into equation (37), we obtain

$$
\frac{V_{0}}{\omega_{n}}=|A| \Omega \sqrt{1-\left(\frac{1}{\alpha}+\frac{k \Delta_{1}}{p_{0}}\right)^{2}}+\sqrt{\frac{2 B^{2}}{1+\cos \pi_{1}}-\left(B-\frac{A}{\alpha}-\frac{\Omega^{2} \Delta_{1}}{1-\Omega^{2}}\right)^{2}} .
$$

Similarly, a dimensionless version of the above equation can be written as

$$
\frac{V_{0}}{B \omega_{d}}=\left|\frac{1}{1-\Omega^{2}}\right| \sqrt{\alpha^{2}-\left(1+\frac{\Delta_{1}}{B}\right)^{2}}+\sqrt{\frac{2}{\Omega^{2}\left(1+\cos \pi_{1}\right)}-\left(\frac{1}{\Omega}-\frac{1}{\Omega\left(1-\Omega^{2}\right)}-\frac{\Omega \Delta_{1}}{B\left(1-\Omega^{2}\right.}\right)^{2}},
$$

where $\Delta_{1} / B$ is the non-dimensionalized deviation.

We first solve equation (46) for $t_{1}$, then formula (47) for $\Delta_{1}$, and finally formula (48) for $V_{0}$. According to $t_{1}$ solved from equation (46) and $t_{2}$ solved from equation (43), we plot the two phase lags $\omega_{d} t_{1}$ and $\omega_{d} t_{2}$ in Figure 5 for $1 / \alpha=0.1,0 \cdot 2,0.3,0.4,0.5$ and 0.6 . The 


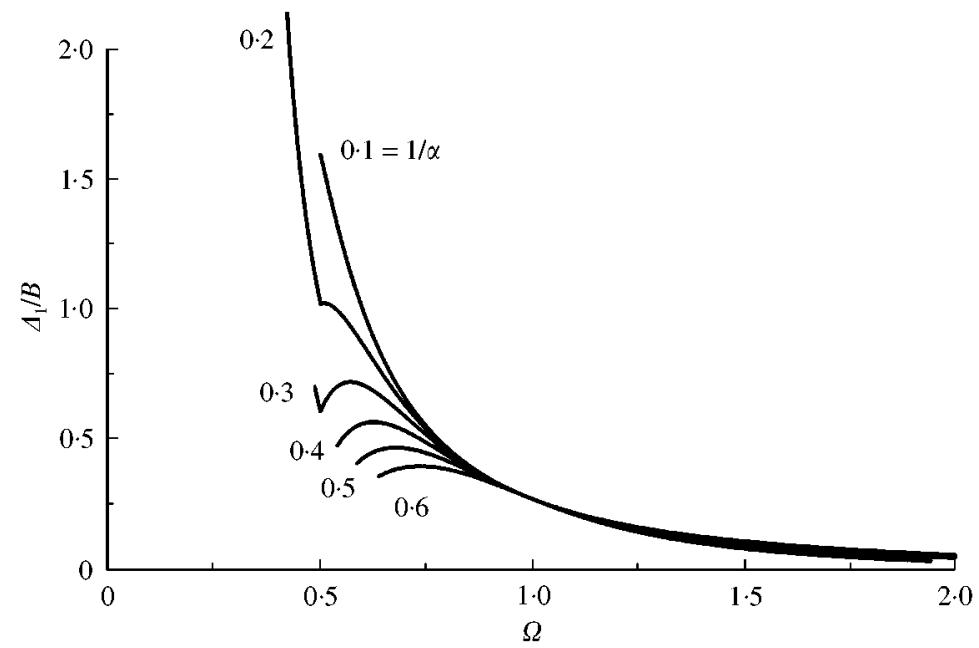

Figure 6. The variation of the dimensionless deviation $\Delta_{1} / B$ with respect to the frequency ratio $\Omega$ for six values of $1 / \alpha$.

dimensionless deviations $\Delta_{1} / B$ are plotted in Figure 6 , which show that the larger the force ratio $\alpha$ is, the larger the deviation $\Delta_{1} / B$ will be in the range when $\Omega<1$. When $\Omega>1$, these curves merge together and tend to zero values. In Figure 7 the results calculated by equation (49) are compared with the exact responses in the non-sticking steady state for the values of $1 / \alpha=0 \cdot 1,0 \cdot 2,0 \cdot 3,0 \cdot 4,0 \cdot 5$ and $0 \cdot 6$. It can be seen that the estimation fits very well with the exact maximum velocity [2].

\section{REMARKS ON DEN HARTOG'S ESTIMATE}

A famous estimate of Den Hartog [1] and Hundal [6] that matches only the following conditions:

$$
x(0)=\Delta_{0}, \quad \dot{x}(0)=0, \quad x\left(\pi / \omega_{d}\right)=-\Delta_{0}, \quad \dot{x}\left(\pi / \omega_{d}\right)=0,
$$

under $p_{0} \cos \left(\omega_{d} t+\phi\right)$ has been proposed by Den Hartog [1] to derive the steady state non-sticking oscillation solutions. There are only two unknowns $\Delta_{0}$ and $\phi$ that are required to be matched. In the lower branch of the phase curve in the plane $(x, \dot{x})$ (see Figure 2), the resulting steady state response was found to be

$$
x(t)=A \cos \left(\omega_{d} t+\phi\right)+B+C_{1} \sin \omega_{n} t+C_{2} \cos \omega_{n} t
$$

in the range $0 \leqslant t \leqslant \pi / \omega_{d}$, where $A$ and $B$ were defined in equations (5) and (6) respectively. Using the first two conditions in equations (50)-(53) he obtained

$$
C_{1}=\Omega A \sin \phi=\frac{-B \sin \pi_{1}}{1+\cos \pi_{1}}, \quad C_{2}=\Delta_{0}-B-A \cos \phi=-B .
$$

Then, by matching the remaining two conditions in equations (50)-(53) the maximum displacement $\Delta_{0}$ was given by equation (42) and it phase lag was $\phi=\omega_{d} t_{2}-\pi / 2$ (and hence its time lag $\left.t_{2}-\pi /\left(2 \omega_{d}\right)\right)$ with $t_{2}$ given by equation (43). It is thus concluded that the new 

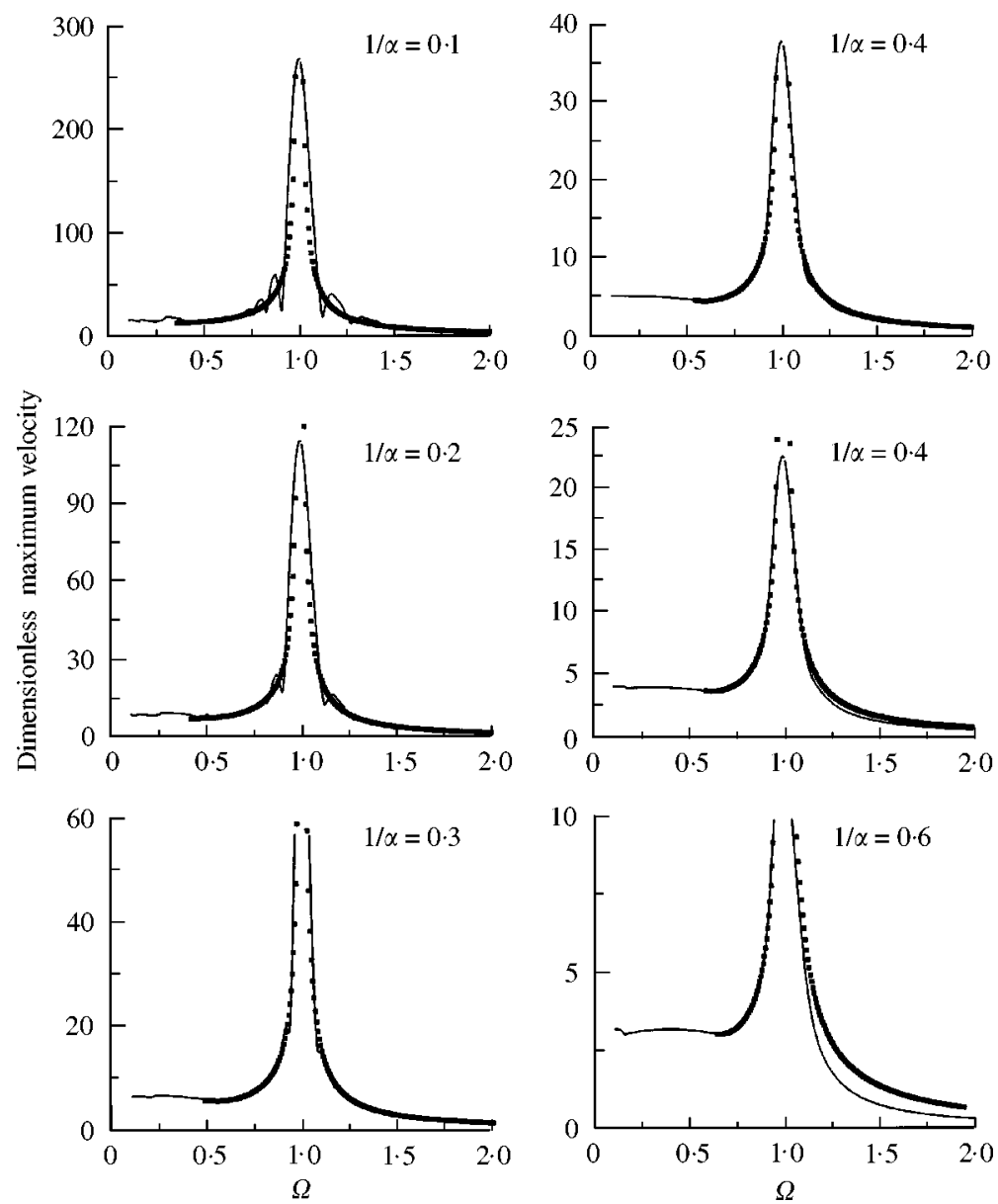

Figure 7. The comparison of the dimensionless maximum velocity between the exact solution and the new estimate for six values of $1 / \alpha$ : - , exact; $\mathbf{\square}$, estimated.

estimate and the estimate of Den Hartog provide the same formulae for the maximum displacement $\Delta_{0}$ and its time lag $t_{2}-\pi /\left(2 \omega_{d}\right)$ (and hence its phase lag $\left.\omega_{d} t_{2}-\pi / 2\right)$, but the new estimate does give additional information about the maximum velocity $V_{0}$ and its time $\operatorname{lag} t_{1}-\pi /\left(2 \omega_{d}\right)$ (and hence its phase lag $\left.\omega_{d} t_{1}-\pi / 2\right)$.

\section{MINIMUM DRIVING AMPLITUDE REQUIRED TO AVOID STICKING}

What is the minimum driving amplitude required to avoid sticking? More precisely, in the parametric space, what is the border line bounding the domain of non-sticking oscillations? Derived below is a precise formula for the border line based on the zero-duration criterion of the sticking phase [2]:

$$
\left|p_{0} \sin \omega_{d} t-k x(t)\right| \geqslant r_{y} .
$$

Accordingly, the border line is determined by the following conditions:

$$
\dot{x}\left(t_{2}\right)=0, \quad\left|p_{0} \sin \omega_{d} t_{2}-k x\left(t_{2}\right)\right|=r_{y} .
$$




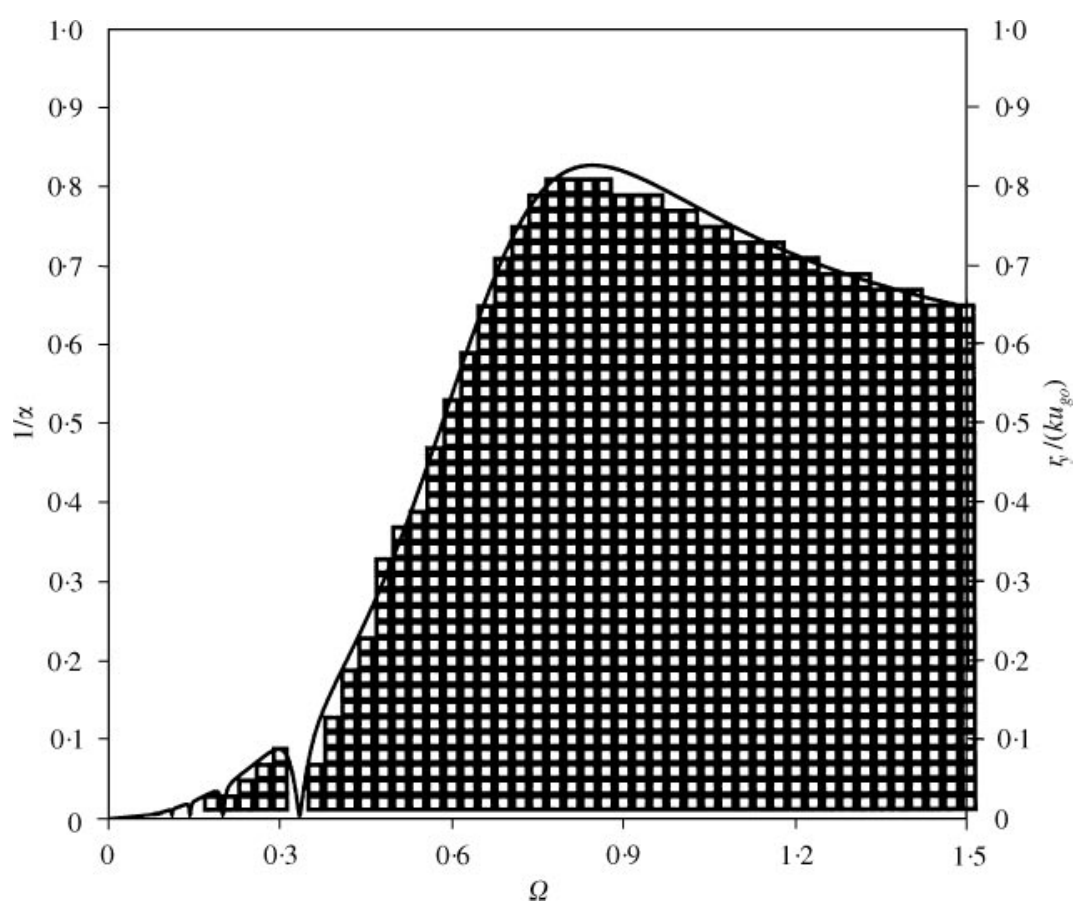

Figure 8. The minimum driving force amplitude required to prevent sticking; the solid curve represents equation (61).

With the aid of equations (17)-(20), (40) and (42) the conditions (58) and (59) are reduced to

$$
B^{2}\left(\left[\frac{\alpha \Omega}{1-\Omega^{2}}\right]^{2}-\left[\frac{\sin \pi_{1}}{1+\cos \pi_{1}}\right]^{2}\right)\left(\frac{k}{r_{y}}-\frac{p_{0}}{r_{y} A}\right)^{2}=\Omega^{2},
$$

which, on using equations (5)-(9), is further refined to

$$
\alpha=\sqrt{\left(\frac{1}{\Omega^{2}}-1\right)^{2}\left[1+\left(\frac{\Omega \sin \pi_{1}}{1+\cos \pi_{1}}\right)^{2}\right]} .
$$

This equation gives a closed-form formula for the border line bounding the domain of steady state non-sticking oscillatory responses in the space of parameters $\alpha$ and $\Omega$. It provides engineers the minimum driving force amplitude required to prevent sticking, for different friction bounds and different frequency ratios. In Figure 8 it is displayed as a solid line; besides, the points of zero stop per cycle obtained from the exact solutions [2] are also displayed for comparison. It can be seen that the above formula gives a very accurate estimation of the border line of the domain of steady state non-sticking oscillatory responses. The value of $\alpha$ which is greater than what the above formula predicts will make the oscillator vibrate without stops in the steady state, so that the criterion for zero stop per cycle is

$$
\alpha \geqslant \sqrt{\left(\frac{1}{\Omega^{2}}-1\right)^{2}\left[1+\left(\frac{\Omega \sin \pi_{1}}{1+\cos \pi_{1}}\right)^{2}\right]}
$$


In passing we note that the rationale for the above derivation is markedly different from that of Den Hartog [1], who derived a formula [equation (21a) therein] to specify the validity domain of non-sticking oscillation motions based on the assumption that $\dot{x}(t)<0$ in the range $0<t<\pi / \omega_{d}$. Thus, this formula depends on time $t$ and hence fails to give a definite estimation of the minimum driving force amplitude to avoid sticking.

\section{SIZE OF THE DISSIPATION LOOP}

For the engineering purposes of dissipating undesirable power through contact friction we now investigate the energy dissipation capacity of the friction oscillator. The size of the energy dissipation loop is

$$
s=4 r_{y} \Delta_{0}
$$

where $\Delta_{0}$ is the maximum displacement in the steady state. Substituting equation (42) for $\Delta_{0}$ into equation (63) and normalizing $s$, we obtain

$$
\Lambda_{1}:=\frac{2 k s}{p_{0}^{2}}=\frac{8}{\alpha\left|1-\Omega^{2}\right|} \sqrt{1-\left(\frac{\left(1-\Omega^{2}\right) \sin \pi_{1}}{\alpha \Omega\left(1+\cos \pi_{1}\right)}\right)^{2}} .
$$

Some remarks on the above formula follow. (1) The left-hand side may be understood as the dissipation per unit elastic energy, since $p_{0} / k$ is the lossless static displacement of the elastic response and $p_{0}^{2} /(2 k)$ is the lossless static elastic energy. (2) The first term on the right-hand side decreases with $\alpha$ for each fixed $\Omega$; conversely, the second term increases with $\alpha$. Therefore, there exists a particular value of the best $\alpha$ to maximize $\Lambda_{1}$ for each fixed $\Omega$; letting $\mathrm{d} \Lambda_{1} / \mathrm{d} \alpha=0$ for each fixed $\Omega$ we can obtain such $\alpha$ and the corresponding maximum of $\Lambda_{1}$ as follows:

$$
\begin{aligned}
\alpha & =\frac{\sqrt{2}\left|\left(1-\Omega^{2}\right) \sin \pi_{1}\right|}{\Omega\left(1+\cos \pi_{1}\right)}, \\
\Lambda_{1}^{\max } & =\frac{4 \Omega\left(1+\cos \pi_{1}\right)}{\left(1-\Omega^{2}\right)^{2}\left|\sin \pi_{1}\right|} .
\end{aligned}
$$

The variations of the above $\alpha$ and $\Lambda_{1}^{\max }$ with respect to $\Omega$ are plotted in Figures 9 (a) and (b) respectively, where only applicable points are shown and non-zero stop points had been excluded by using equation (62). Now, we are in a good position to assess the influence of the control parameter $\alpha$ on $\Lambda_{1}$. Equation (64) is used to investigate the variation of the non-dimensionalized size of the dissipation loop with respect to $\alpha$, when $k, p_{0}$ and $\Omega$ are fixed, that is, the influence of the friction bound $r_{y}$ on the size of the dissipation loop. In Figure 10(a) the variation of $\Lambda_{1}$ with respect to $1 / \alpha$ is plotted for $\Omega=1 \cdot 2,1 \cdot 3,1 \cdot 4,1 \cdot 5,1 \cdot 6,1 \cdot 7$ and $1 \cdot 8$. For energy dissipation purposes we usually choose the best $\alpha$ to maximize the dissipation loop size. Under this $\alpha$ the friction oscillator will achieve the best performance of dissipating as much energy as it can.

Similarly, in order to account for the influence of the stiffness $k$ on the size of the dissipation loop when $m, p_{0}, \omega_{d}$ and $\alpha$ are fixed, we may introduce the following non-dimensionalized size of the dissipation loop:

$$
\Lambda_{2}:=\frac{2 m \omega_{d}^{2} s}{p_{0}^{2}}=\frac{8 \Omega^{2}}{\alpha\left|1-\Omega^{2}\right|} \sqrt{1-\left(\frac{\left(1-\Omega^{2}\right) \sin \pi_{1}}{\alpha \Omega\left(1+\cos \pi_{1}\right)}\right)^{2}} .
$$



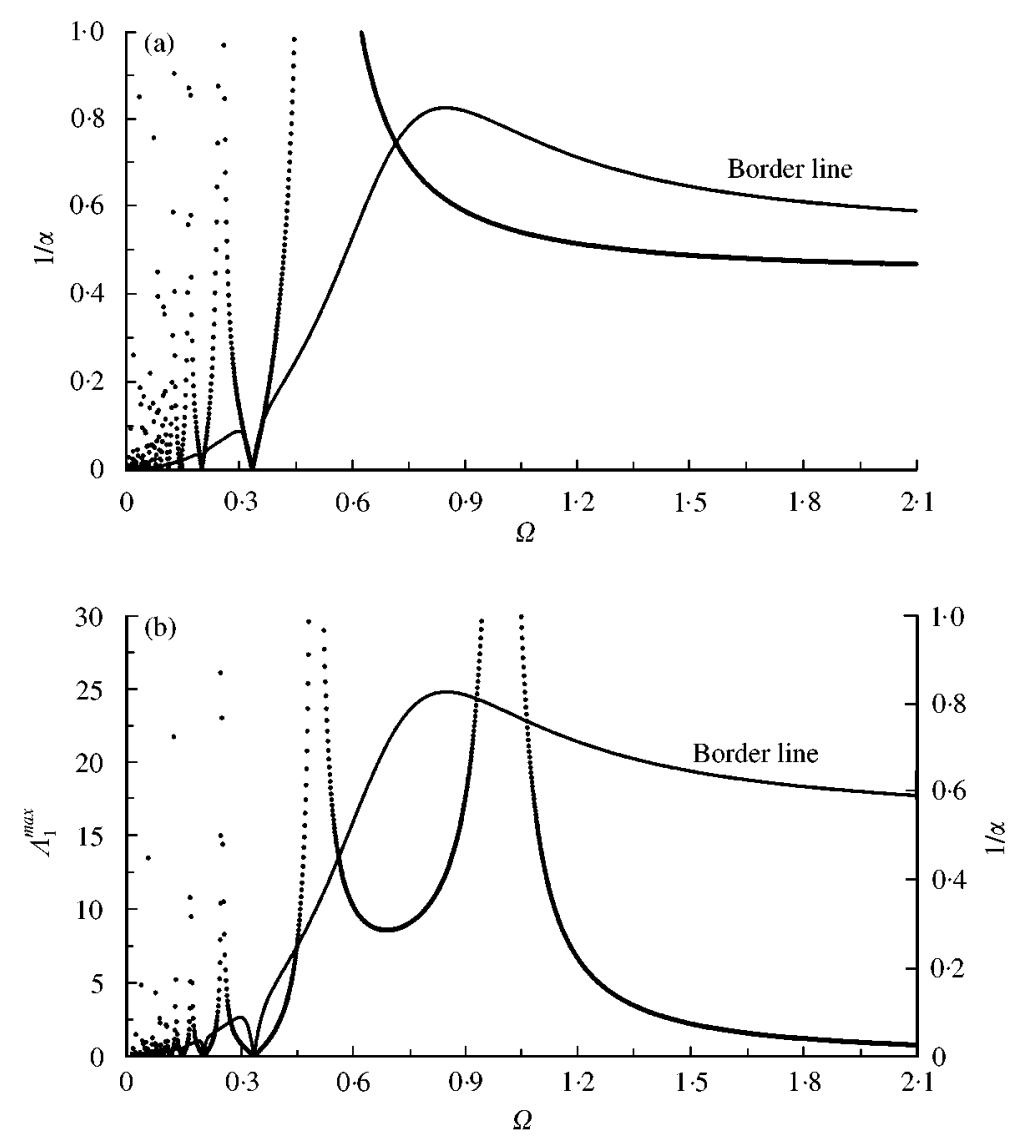

Figure 9. (a) The variation of the best $\alpha$ with respect to $\Omega$. (b) The variation of the maximum dissipation loop size with respect to $\Omega$.

Let $m \omega_{d}^{2}=: k_{d}$ be the pseudo-elastic stiffness. The left-hand side of the above equation may be written as $s /\left(p_{0}^{2} /\left(2 k_{d}\right)\right)$, which, corresponding to (1) in section 8 , may be deemed as the dissipation per lossless static pseudo-elastic energy. Equation (67) is used to investigate the variation of the non-dimensionalized size of the dissipation loop with respect to $\Omega$, when $m, p_{0}, \omega_{d}$ and $\alpha$ are fixed, that is, the influence of the elastic stiffness $k$ on the dissipation loop. In Figure 10(b) the variation of $\Lambda_{2}$ with respect to $\Omega$ is plotted for $1 / \alpha=0 \cdot 1,0 \cdot 2,0 \cdot 3,0 \cdot 4$ and $0 \cdot 5$.

\section{CONCLUDING REMARKS}

A new estimate was developed for the steady state non-sticking oscillatory responses. The resulting formulae (42) and (43) can be used to determine the maximum displacement and its time lag $t_{2}-\pi /\left(2 \omega_{d}\right)$. Since the maximum velocity point in the phase plane may deviate from the velocity axis with a deviation $\Delta_{1}$, which increases when the force ratio $\alpha$ decreases, we proposed equations (48) and (46) for the estimation of the maximum velocity and its time lag $t_{1}-\pi /\left(2 \omega_{d}\right)$. It supplied very good results when compared with the exact maximum velocities. The formulae for the maximum size of the dissipation loop were derived. It is found that for a fixed frequency ratio there exists a value of the best driving force amplitude to maximize the dissipation loop size. The closed-form formula (61) of the border line of 

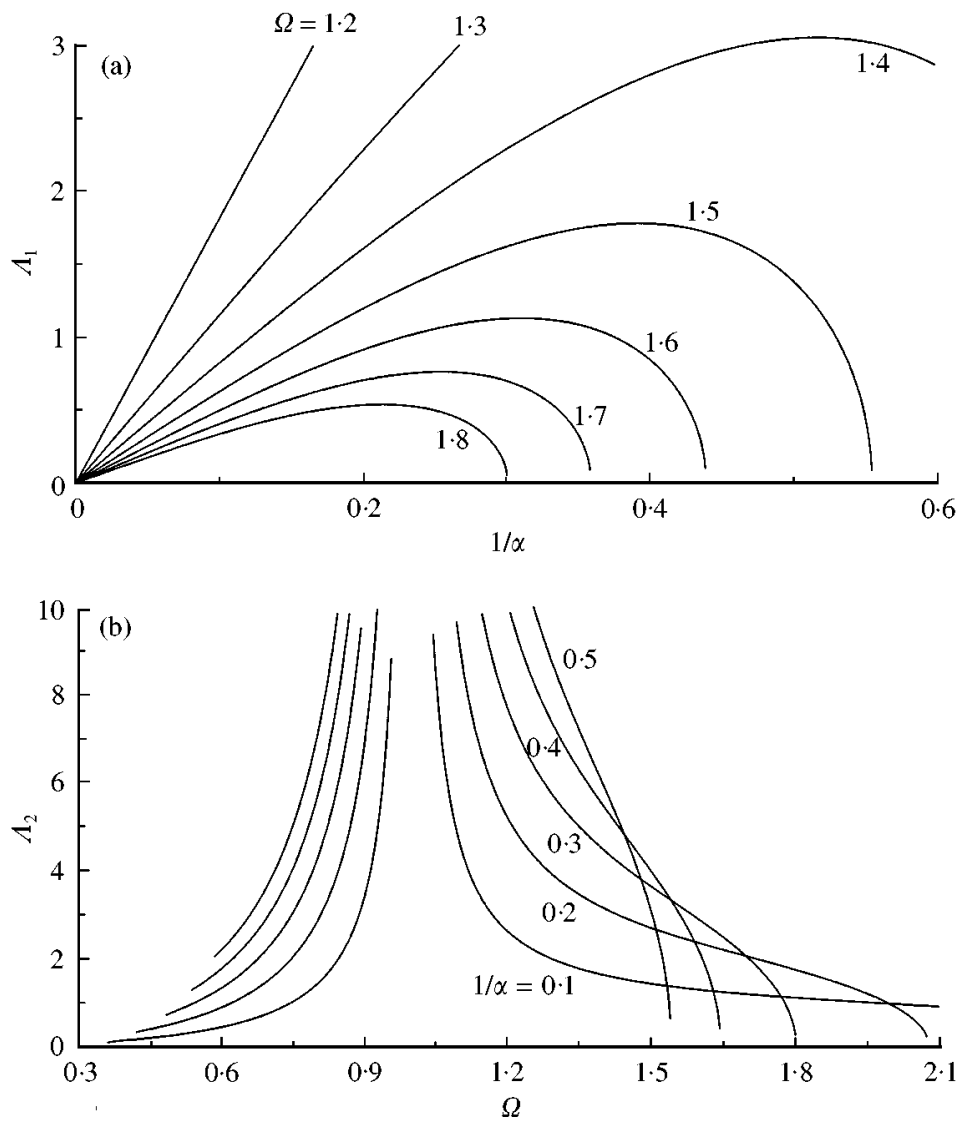

Figure 10. (a) The variation of $\Lambda_{1}$ with respect to $1 / \alpha$ for seven values of $\Omega$. (b) The variation of $\Lambda_{2}$ with respect to $\Omega$ for five values of $1 / \alpha$.

non-sticking oscillation motions was derived. In view of the detrimental effect of sticking on the operation and performance of oscillators, this simple formula must be very useful for engineers in selecting a minimum driving force amplitude to prevent an oscillating object from sticking to the friction surface.

\section{REFERENCES}

1. J. P. Den Hartog 1931 Transactions of the American Society of Mechanical Engineers 53, 107-115. Forced vibrations with combined Coulomb and viscous friction.

2. H.-K. HoNG and C.-S. LIU 2000 Journal of Sound and Vibration 229, 1171-1192. Coulomb friction oscillator: modelling and responses to harmonic loads and base excitations.

3. R. A. Ibrahim 1994 Applied Mechanics Review 47, 209-253. Friction-induced vibration, chatter, squeal, and chaos. Part I: mechanics of contact and friction, Part II: dynamics and modeling.

4. B. Feeny, A. Guran, N. Hinrichs and K. Popp 1998 Applied Mechanics Review 51, 321-341. A historical review on dry friction and stick-slip phenomena.

5. N. Makris and M. C. Constantinou 1991 Mechanics of Structure and Machine 19, 477-500. Analysis of motion resisted by friction. I. Constant Coulomb and linear Coulomb friction.

6. M. S. Hundal 1979 Journal of Sound and Vibration 64, 371-378. Response of a base excited system with Coulomb and viscous friction. 
7. L. Y. Chen, J. T. Chen, C. H. Chen and H.-K. Hong 1994 Mechanics Research Communications 21, 599-604. Free vibration of a sdof system with hysteretic damping.

8. C. Pierre, A. A. Ferri and E. H. Dowell 1985 American Society of Mechanical Engineers Journal of Applied Mechanics 52, 958-964. Multi-harmonic analysis of dry friction damped systems using an incremental harmonic balance method.

9. A. H. NAYFEH and D. T. Mook 1979 Nonlinear Oscillations. New York: Wiley.

10. K. E. BeUCKE and J. M. Kelly 1985 International Journal of Non-Linear Mechanics 23, 211-238. Equivalent linearizations for practical hysteretic systems.

11. B. Westermo and F. UdWAdia 1983 Earthquake Engineering and Structural Dynamics 11, 135-146. Periodic response of a sliding oscillator system to harmonic excitation.

12. N. Mostaghel, M. Hejazi and J. TAnbaKuchi 1983 Earthquake Engineering and Structural Dynamics 11, 355-366. Response of sliding structures to harmonic support motion.

13. R. PARNES 1984 Journal of Sound and Vibration 94, 469-482. Response of an oscillator to a ground motion with Coulomb friction slippage.

14. C. J. Younis and I. G. TADJBAKHSH 1984 American Society of Civil Engineers Journal of Engineering Mechanics 110, 417-432. Response of sliding rigid structure to base excitation.

15. E. MARUi and S. KATO 1984 American Society of Mechanical Engineers Journal of Dynamic Systems, Measurement, and Control 106, 280-285. Forced vibration of a base-excited single-degree-of-freedom system with Coulomb friction.

16. K. Matsui, M. IURA, T. SASAKi and I. KosaKa 1991 Earthquake Engineering and Structural Dynamics 20, 683-697. Periodic response of a rigid block resting on a footing subjected to harmonic excitation.

17. D. S. LARSON and A. FAFITIS 1995 American Society of Civil Engineers Journal of Engineering Mechanics 121, 1226-1233. Periodic response and stability of rigid mass resting on friction-damped sdof oscillator. 